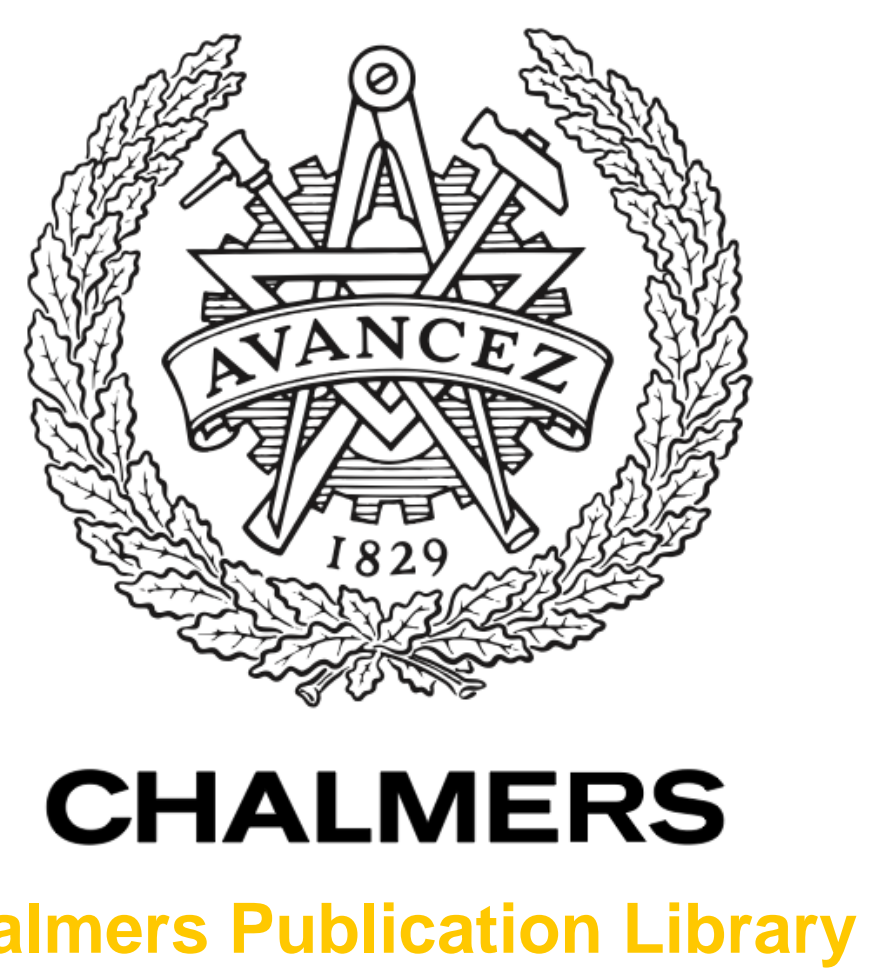

Chalmers Publication Library

\title{
Throughput Analysis for Multi-Point Joint Transmission with Quantized CSI Feedback
}

This document has been downloaded from Chalmers Publication Library (CPL). It is the author's version of a work that was accepted for publication in:

Vehicular Technology Conference (VTC Fall) (ISSN: 1090-3038)

Citation for the published paper:

Makki, B. ; Li, J. ; Eriksson, T. (2012) "Throughput Analysis for Multi-Point Joint

Transmission with Quantized CSI Feedback". Vehicular Technology Conference (VTC Fall)

pp. 1 - 5 .

http://dx.doi.org/10.1109/VTCFall.2012.6399362

Downloaded from: http://publications.lib.chalmers.se/publication/167889

Notice: Changes introduced as a result of publishing processes such as copy-editing and formatting may not be reflected in this document. For a definitive version of this work, please refer to the published source. Please note that access to the published version might require a subscription. 


\title{
Throughput Analysis for Multi-Point Joint Transmission with Quantized CSI Feedback
}

\author{
Behrooz Makki, Jingya Li, Thomas Eriksson and Tommy Svensson \\ Department of Signals and Systems, Chalmers University of Technology, Gothenburg, Sweden \\ Email: behrooz.makki@chalmers.se,jingya.li@chalmers.se, thomase@chalmers.se, tommy.svensson@chalmers.se
}

\begin{abstract}
This paper addresses the problem of limited CSI feedback in coordinated multi-point (CoMP) networks. Specifically, the system throughput is obtained for block-fading CoMP channels with quantized CSI feedback, and the effect of feedback bit allocation on the system throughput is investigated for different user locations and fading distributions. The analytical and simulation results show that substantial throughput increment is achieved via CoMP joint transmission with very limited number of feedback bits per base station. The effect of optimal bit allocation becomes more important for the user that is located in the CoMP cluster edge areas. Also, the standard Zonal-sampling scheme provides the best bit allocation strategy in many cases, maximizing the system throughput.
\end{abstract}

\section{INTRODUCTION}

Recently, coordinated multi-point (CoMP) joint transmission, also known as network multiple-input-multiple-output (MIMO), is considered as a promising technique to mitigate inter-cell interference (ICI) and improve the spectral efficiency [1], [2]. In CoMP systems, coordinated base stations (BSs) are inter-connected via a high-speed backbone. In the case of joint transmission, the coordinated BSs share channel state information (CSI) and the user data. Hence, the ICI is reduced by exploiting the signals transmitted from other cells to assist the transmission rather than treating them as interference. This will lead to considerable improvement in throughput, particularly for cell-edge users where the improvements are most needed.

A major drawback of multi-point joint transmission is, however, the enormous amount of CSI feedback overhead required at the coordinated BSs. In order to make the feedback overhead affordable, clustering of BSs, i.e., dividing the network into small subsystems or clusters of coordinated BSs, has been considered in [3]-[5]. In [6], a threshold based approach is proposed which allows the receiver not to feedback CSI of BSs with weak channel quality inside a cooperation cluster. In [7], quantized CSI feedback of a subset of BSs is considered for the downlink multi-user network MIMO system.

In this paper, we address the problem of quantized CSI feedback in the downlink of a CoMP cluster, consisting of multiple BSs and one user. In contrast to, e.g., [3]-[6], where no realistic quantized CSI feedback and quantization bit allocation were considered, this paper concentrates on the optimal quantization boundaries and the reconstruction points of the quantizers maximizing the system throughput. Moreover, we take the data outage probability, due to imperfect CSI at the transmitter, into account which was not considered by [7]. In this way, the optimal feedback bit allocation between the BSs can be studied, which was not investigated in [3]-[7].

Our scheme works as follows. Assume both the data and the quantized CSI feedback of the user are shared in real time among all BSs in the cluster. The CSI feedback is represented via limited number of feedback bits. Based on the quantization indices, all BSs of the cluster provide non-coherent joint transmission to the user at each time slot. System throughput is analyzed for joint transmission with quantized CSI feedback taking user data decodability into account. Further, the effect of bit allocation on the system throughput is investigated for different user locations and fading distributions.

Analytical and simulation results show that considerable system throughput improvement is achieved via base stations cooperation with limited number of feedback bits per base station. The effect of optimal bit allocation becomes more important for the user located in the CoMP cluster edge areas. In harmony with practical schemes [8], the standard Zonal sampling scheme provides the best bit allocation strategy in many cases, maximizing the system throughput. Moreover, considering quantized CSI, the maximum throughput is obtained when the data is transmitted with a rate corresponding to the worst (lowest) gains within the quantization regions. The results should be relevant for researchers involved in the evolution of $4 \mathrm{G}$ systems research and standardization, such as evolution of the WINNER 4G system concept [9] or the Long Term Evolution (LTE) standard by the 3rd Generation Partnership Project (3GPP) [10], where partial CSI is one of the most important issues.

The rest of the paper is organized as follows. In Section II, we present the system model. In Section III, the system throughput with quantized CSI feedback is analyzed for multipoint non-coherent joint transmission. Section IV presents the simulation results with respect to different bit allocation strategies and user locations. Conclusions are presented in Section V.

\section{SYSTEM MODEL}

As illustrated in Fig.1, we consider a communication setup with $N$ BSs and one user. The BSs are assumed to have full cooperation, provide joint transmission to the user and constrained to have the same limited transmission power $P$. In this way, the channel can be modeled as

$$
Y=\sum_{n=1}^{N} l_{n} H_{n} X_{n}+Z
$$




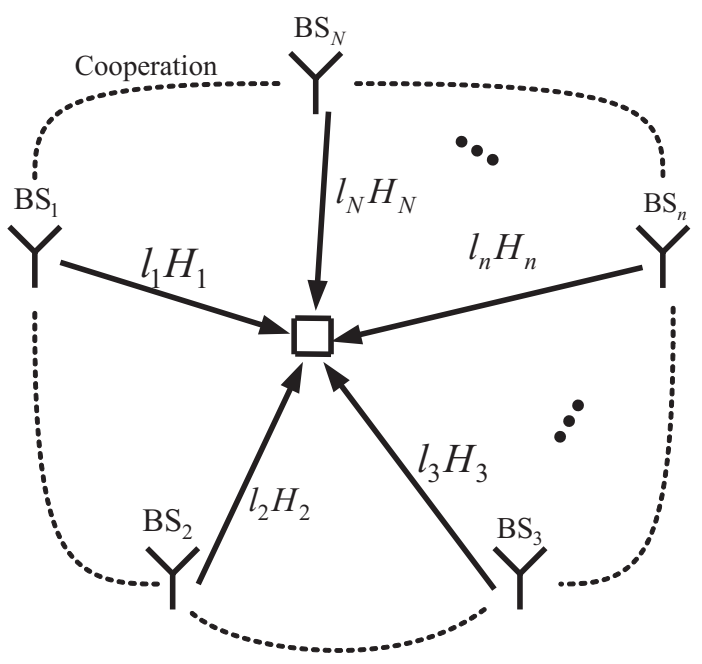

Figure 1. System model. The network consists of $N$ base stations (BSs) and one user. The base stations have full cooperation with each other. Further, while the receiver has perfect knowledge about the channels quality, the BSs are provided with quantized CSI represented via limited number of feedback bits. Considering block fading channels, the system throughput is investigated under different bit allocation strategies.

where $Y$ denotes the received signal, $l_{n}$ is the long-term channel gain between the $n$-th $\mathrm{BS}$ and the user, consisting of path loss and shadow fading, $X_{n}$ is the data transmitted from the $n$-th $\mathrm{BS}$ and $H_{n}$ represents the independent and identically distributed (iid) small-scale fast fading variable between the $n$-th $\mathrm{BS}$ and the user. Also, $Z \sim \mathcal{C} \mathcal{N}\left(0, N_{0}\right)$ denotes additive iid complex Gaussian noise plus the interference received from the other base stations/clusters, where we set $N_{0}=1$. Moreover, we define $G_{n}=\left|H_{n}\right|^{2}$ which is denoted the $n$-th channel gain in the following. Although the results are valid also if the small-scale fast fading channels have different distributions, for simplicity we assume that they experience the same probability density function (pdf). Then, this scenario can be, with no loss of generality, mapped into the case where the channels experience identical pdfs but the BSs have different power constraints $\left|X_{n}\right|^{2} \leq P_{n}$, where $P_{n}=P\left|l_{n}\right|^{2}, n=1, \ldots, N$. Finally, the gain pdf:s are represented by $f_{G_{n}}(g), n=1, \ldots, N$.

We focus on block-fading channels ${ }^{1}$ where the channel gains remain constant for a long duration, generally determined by the channel coherence time, and then change independently according to the fading pdfs. Then, motivated by the transmission of training sequences, it is assumed that there is perfect instantaneous knowledge about the channels gains at the receiver, which is an acceptable assumption under blockfading conditions [13]-[18]. On the other hand, due to limited feedback resources, the BSs are provided with quantized CSI about the channel gains represented via limited number of feedback bits.

Evaluation yardstick: Our figure of merit is the system throughput. Let $\tau_{k}$ be the number of channel uses in the $k$ th time slot. Then, the total number of channel uses and the total number of successfully decoded information nats ${ }^{2}$ up to

\footnotetext{
${ }^{1}$ As discussed in [11], [12], the information theoretic results of block-fading channels match the results of actual codes for practical code lengths (e.g., $\simeq 100$ channel uses).

${ }^{2}$ If not specified, the results are presented in natural logarithm basis. Further in all simulations the throughput is presented in nats-per-channel-use (npcu).
}

the end of the $k$-th time slot are denoted by $\tau^{(k)}$ and $Q^{(k)}$, respectively. In this way, the system throughput is defined as

$$
\eta \doteq \lim _{k \rightarrow \infty} \frac{Q^{(k)}}{\tau^{(k)}}=\frac{E\{Q\}}{E\{\tau\}}
$$

where $Q$ is the number of successfully decoded information nats in each transmission, $\tau$ is the number of channel uses in a data transmission time slot and $E\{$.$\} represents the expectation$ operator [19]. Here, we focus on fixed-length coding schemes. Therefore, defining $R\left(g_{1}, \ldots, g_{N}\right)$ as the achievable rate for gain realizations $g_{n}, n=1, \ldots, N$ and $L_{\mathrm{c}}$ (in channel uses) as the codewords length, the system throughput is simplified to

$$
\eta=\frac{E\left\{L_{c} R\left(g_{1}, \ldots, g_{N}\right)\right\}}{L_{c}}=E\left\{R\left(g_{1}, \ldots, g_{N}\right)\right\}=\bar{R},
$$

that is, the channel average rate [17]-[20]. In the following, we evaluate the effect of quantized CSI and bit allocation on the system throughput.

\section{SYSTEM THROUGHPUT WITH QUANTIZED CSI FEEDBACK}

At the beginning of each fading block, the receiver implements $N$ deterministic quantization functions

$$
C_{n}\left(g_{n}\right)=i_{n} \text { if } g_{n} \in S_{i_{n}}=\left[\tilde{g}_{i_{n}-1}, \tilde{g}_{i_{n}}\right), i_{n}=1, \ldots, I_{n}
$$

which partitions the gain pdf of the $n$-th link, $n=1, \ldots, N$, into $I_{n}$ regions $S_{i_{n}}, i_{n}=1, \ldots, I_{n}$. Here, $\tilde{g}_{i_{n}}$ 's denote the quantization boundaries where we have $\tilde{g}_{0}=0, \tilde{g}_{I_{n}}=\infty$. Also, $\pi_{i_{n}}=\operatorname{Pr}\left\{g_{n} \in S_{i_{n}}\right\}=\int_{\tilde{g}_{i_{n}-1}}^{\tilde{g}_{i_{n}}} f_{G_{n}}(g) \mathrm{d} g$ represents the probability that the $n$-th channel gain falls into the region $S_{i_{n}}$. Then, the quantization indices are fed back to all BSs. Therefore, assuming unicast to each BS, the total number of feedback bits per block would be

$$
B=\sum_{n=1}^{N} b_{n}, b_{n}=\left\lfloor\log _{2} I_{n}\right\rfloor+1
$$

where $\lfloor$.$\rfloor denotes the lower integer value.$

Getting the quantization indices $\left\{i_{n}, n=1, \ldots, N\right\}$, some codeword is created by cooperation of the BSs such that $Q_{i_{1} \ldots i_{N}}$ information nats is sent to the receiver. Therefore, as the length of the codewords is $L_{\mathrm{c}}$, the transmission rate would be $R_{i_{1} \ldots i_{N}}=\frac{Q_{i_{1} \ldots i_{N}}}{L_{\mathrm{c}}}$. It is obvious that, based on the received quantization indices $\left\{i_{n}, n=1, \ldots, N\right\}$, the transmission rates are selected such that ${ }^{3}$

$$
\log \left(1+\sum_{n=1}^{N} \tilde{g}_{i_{n}-1} P_{n}\right) \leq R_{i_{1} \ldots i_{N}}<\log \left(1+\sum_{n=1}^{N} \tilde{g}_{i_{n}} P_{n}\right),
$$

that is, the rates are selected to be some value between the two extreme possible transmission rates for the given set of quantization indices. The data is successfully decoded by the receiver if the channel gains support the rate, i.e., $R_{i_{1} \ldots i_{N}} \leq \log \left(1+\sum_{n=1}^{N} g_{n} P_{n}\right)$, where $\gamma=\sum_{n=1}^{N} g_{n} P_{n}$ is the instantaneous signal-to-interference-and-noise ratio (SINR)

\footnotetext{
${ }^{3}$ In (6), we have used the fact that with an equivalent SINR $x$ the maximum decodable rate is $\log (1+x)$ if the length of the codewords is (infinitely) long. For systems with finite code lengths, the achievable rate would be less.
} 
at the receiver. Hence, for a set of quantization indices $\left\{i_{n}, n=1, \ldots, N\right\}$, the expected transmission rate is

$$
\begin{aligned}
\bar{R}_{i_{1} \ldots i_{N}}= & R_{i_{1} \ldots i_{N}} \beta_{i_{1} \ldots i_{N}} \\
\beta_{i_{1} \ldots i_{N}}= & \operatorname{Pr}\left\{R_{i_{1} \ldots i_{N}} \leq\right. \\
& \left.\log \left(1+\sum_{n=1}^{N} g_{n} P_{n}\right) \mid g_{n} \in S_{i_{n}}, n=1, \ldots, N\right\}
\end{aligned}
$$

where $\beta_{i_{1} \ldots i_{N}}$ is the probability that the data with rate $R_{i_{1} \ldots i_{N}}$ is decodable if the channels are in the regions $S_{i_{n}}, n=$ $1, \ldots, N$.

In this way, considering all quantization regions, the system throughput is obtained by

$$
\begin{aligned}
\eta & =\sum_{i_{1}=1}^{I_{1}} \ldots \sum_{i_{N}=1}^{I_{N}} \operatorname{Pr}\left\{g_{n} \in S_{i_{n}}, n=1, \ldots, N\right\} \bar{R}_{i_{1} \ldots i_{N}} \\
& =\sum_{i_{1}=1}^{I_{1}} \ldots \sum_{i_{N}=1}^{I_{N}}\left(\prod_{n=1}^{N} \pi_{i_{n}}\right) \bar{R}_{i_{1} \ldots i_{N}} \\
& =\sum_{i_{1}=1}^{I_{1}} \ldots \sum_{i_{N}=1}^{I_{N}} R_{i_{1} \ldots i_{N}} \Upsilon_{i_{1} \ldots i_{N}}
\end{aligned}
$$

where we have

$$
\begin{aligned}
& \Upsilon_{i_{1} \ldots i_{N}}=\left(\prod_{n=1}^{N} \pi_{i_{n}}\right) \beta_{i_{1} \ldots i_{N}}= \\
& \int_{\tilde{g}_{i_{1}-1}}^{\tilde{g}_{i_{1}}} . . \int_{\tilde{g}_{i_{N}-1}}^{\tilde{g}_{i_{N}}}\left(\prod_{n=1}^{N} f_{G_{n}}\left(g_{n}\right)\right) \mathbf{1}\left(g_{1}, \ldots, g_{N} ; R_{i_{1} \ldots i_{N}}\right) \mathrm{d} g_{1} \ldots \mathrm{d} g_{N} .
\end{aligned}
$$

Here, $\mathbf{1}\left(x_{1}, \ldots, x_{N} ; R_{i_{1} \ldots i_{N}}\right)$ is an indicator function defined as

$\mathbf{1}\left(g_{1}, \ldots, g_{N} ; R_{i_{1} \ldots i_{N}}\right)=\left\{\begin{array}{l}1 \text { if } \log \left(1+\sum_{n=1}^{N} g_{n} P_{n}\right) \geq R_{i_{1} \ldots i_{N}} \\ 0 \text { otherwise }\end{array}\right.$

In the following, we show that the maximum throughput is achieved when the transmission rate $R_{i_{1} \ldots i_{N}}$ is selected to be the value corresponding to the worst (lowest) gains within quantization regions $S_{i_{n}}, i_{n}, n=1, \ldots, N$ (except the cases where the channels are in their first quantization regions).

Theorem 1: The maximum system throughput is achieved by $R_{i_{1} \ldots i_{N}}=\log \left(1+\sum_{n=1}^{N} \tilde{g}_{i_{n}-1} P_{n}\right), i_{n} \neq 0, n=1, \ldots, N$.

Proof: Let us assume that $R_{i_{1} \ldots i_{N}}>\log (1+$ $\left.\sum_{n=1}^{N} \tilde{g}_{i_{n}-1} P_{n}\right)$. Then, if the channel gains are such that $\log \left(1+\sum_{n=1}^{N} g_{n} P_{n}\right)<R_{i_{1} \ldots i_{N}}$, the data transmitted with rate $R_{i_{1} \ldots i_{N}}$ is lost, that is, outage happens. However, by increasing the quantization boundaries $\tilde{g}_{i_{n}-1}$ such that $R_{i_{1} \ldots i_{N}}=\log (1+$ $\left.\sum_{n=1}^{N} \tilde{g}_{i_{n}-1} P_{n}\right)$ we increase the contribution of this rate on the throughput from zero to $\log \left(1+\sum_{n=1}^{N} \tilde{g}_{i_{n}-1} P_{n}\right)$ and, consequently, increase the system throughput. Therefore, in the optimal case we have $R_{i_{1} \ldots i_{N}}=\log \left(1+\sum_{n=1}^{N} \tilde{g}_{i_{n}-1} P_{n}\right)$. Note that, according to (6), this is the lowest possible rate that can be considered for a given set of quantization indices.

Using the theorem, the other parameters such as the quantization boundaries and the transmission rates for the case where the channels fall in the first quantization regions can be optimized, in terms of throughput, numerically. Finally, it is worth noting that considering a single base station, the theorem is reduced to the results obtained in [20].
Based on the theorem, the system throughput, i.e., (8), is simplified to

$$
\begin{aligned}
\eta & =\sum_{i_{2}=1}^{I_{2}} \ldots \sum_{i_{N}=1}^{I_{N}} R_{1 i_{2} \ldots i_{N}} \Upsilon_{1 i_{2} \ldots i_{N}} \\
& +\ldots+\sum_{i_{1}=1}^{I_{1}} \ldots \sum_{i_{N-1}=1}^{I_{N-1}} R_{i_{1} \ldots i_{N-1} 1} \Upsilon_{i_{1} \ldots i_{N-1} 1} \\
& +\sum_{\substack{\forall i_{n}, n=1, \ldots, N \\
i_{n} \neq 1}} \ldots \sum_{n=1}\left(\prod_{n=1}^{N} \pi_{i_{n}}\right) \log \left(1+\sum_{n=1}^{N} \tilde{g}_{i_{n}-1} P_{n}\right) .
\end{aligned}
$$

The first two lines in (11) are for the cases where one of the channels falls into its first quantization region. Further, the last term is for the case where none of the gains are in the first quantization region, where Theorem 1 is valid. Also, it is interesting to note that, based on Theorem 1, the outage may happen if and only if one of the channels is in its first quantization region. Otherwise, as the codewords are (asymptotically) long, the data is always decoded with probability of 1 . Finally, assuming infinitely many quantization regions for each channel, i.e., $I_{n} \rightarrow \infty, n=1, \ldots, N$, (11) is simplified to

$$
\eta=\int_{0}^{\infty} \ldots \int_{0}^{\infty}\left(\prod_{n=1}^{N} f_{G_{n}}\left(g_{n}\right)\right) \log \left(1+\sum_{n=1}^{N} g_{n} P_{n}\right) \mathrm{d} g_{1} \ldots \mathrm{d} g_{N}
$$

which is the system throughput with perfect CSI at the BSs. In the following, we study (11) in more details where the system throughput is investigated for different user locations and small-scale fast fading distributions.

\section{Simulation RESUlts}

Simulation results are presented for Rayleigh-fading and channels with Nakagami-2 gain distributions respectively given by

$$
f_{G}(g)=\lambda e^{-\lambda g}, g \geq 0
$$

and

$$
f_{G}(g)=\frac{8}{\lambda^{2}} g^{3} e^{-\frac{2}{\lambda} g^{2}}, g \geq 0
$$

where we set $\lambda=1$. Note that Rayleigh-fading channels are good models for tropospheric and ionospheric signal propagation as well as the effect of heavily built-up urban environments on radio signals [21], [22]. On the other hand, as discussed in, e.g., [23], [24], Nakagami distributions are appropriate models for the cases where the fading is not so severe.

Notice that, as described in the system model, the effect of user location can be mapped into different transmit power constraints of BSs. Considering three base stations with different mapped transmission powers, Figs. 2 and 3 respectively demonstrate the system throughput for Rayleighfading and Nakagami channels as a function of number of feedback bits. Further, the system throughput with perfect CSI at the BSs, i.e., (12), is considered as the system performance upper bound. In each point, the triple $\left(b_{1}, b_{2}, b_{3}\right)$ shows the optimal bit allocation maximizing the system throughput, i.e., (11). These values have been determined by exhaustive search among all possible bit allocation strategies. Then, Fig. 4 studies the effect of BSs distances from the user on the system 


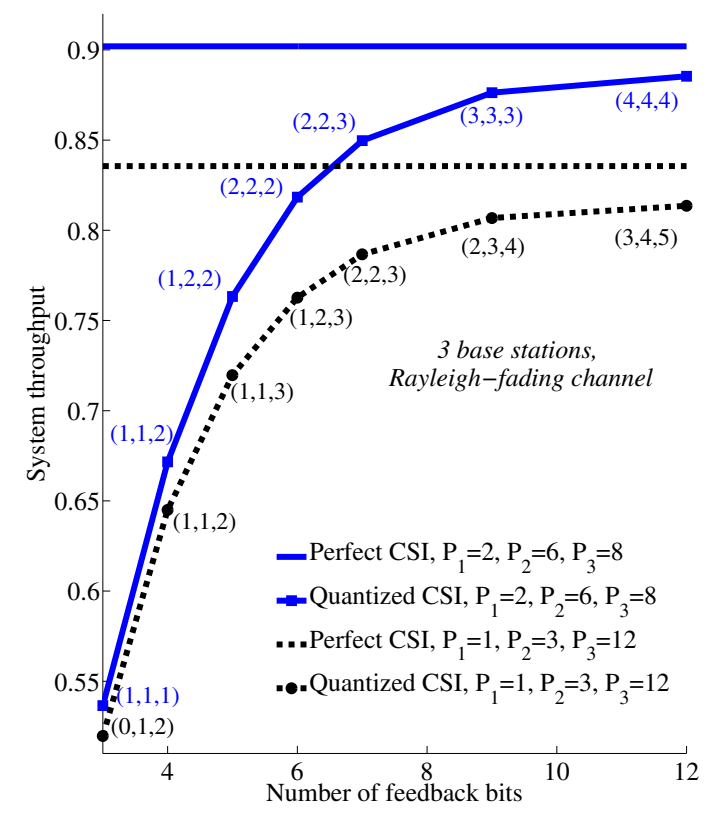

Figure 2. System throughput vs number of feedback bits, 3 base stations, Rayleigh fading channel. The triple $\left(b_{1}, b_{2}, b_{3}\right)$ represents the number of feedback bits optimally distributed between the base stations.

throughput. For this reason, a distance factor $\alpha$ is considered where, while the first BS power is kept fixed, the other BSs powers change as $\left(P_{2}, P_{3}\right)=\left(\frac{32}{3}-\alpha, \alpha\right), \alpha \in\left[0, \frac{32}{3}\right]$. Finally, in order to evaluate the effect of base stations cooperation, Fig. 5 compares the results with the case where the data is transmitted from a single base station. To have a fair comparison, the number of quantization bits and the transmission power of the single transmitter are assumed to be the sum of the three cooperative base stations feedback bits and powers.

Simulation results show that:

- Considerable performance improvement can be achieved by using joint transmission CoMP with limited number of feedback bits, particularly when the fading severity is not too high (Nakagami distribution, Fig. 3). For instance, for both Nakagami and Rayleigh-fading channels, 95 percent of the system full-CSI throughput is reached by 3 bits per base station feedback.

- The effect of optimal bit allocation becomes more important when the user experiences different channel qualities from the BSs, i.e., the user is close to the CoMP cluster edge area (Figs. 2 and 3, for the case of $P_{1}=1, P_{2}=$ $3, P_{3}=12$ ). However, this effect diminishes when the channel qualities are the same, that is, the user is close to the CoMP cluster center area.

- Higher system throughput is achieved when the channel qualities are (almost) the same (Figs. 2-4). This point emphasizes the positive effect of power allocation on the system performance. Specifically, the channel inversion technique [11], where the powers are distributed such that the base stations have the same contribution on the received SINR, seems to be efficient.

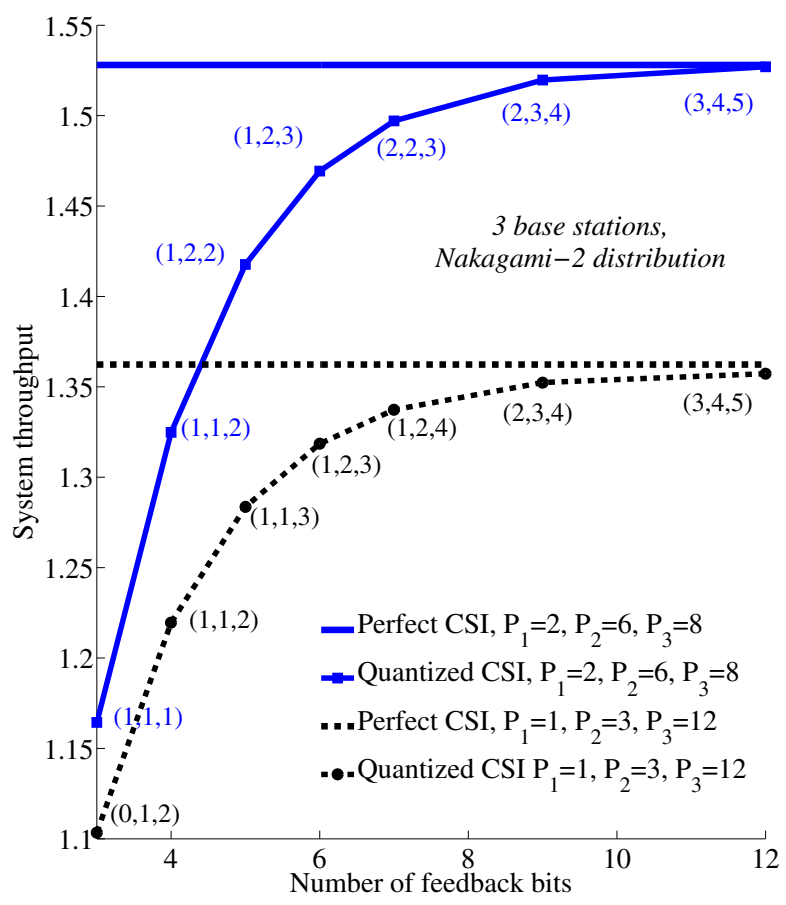

Figure 3. System throughput vs number of feedback bits, 3 base stations, Nakagami-2 gain distributions. The triple $\left(b_{1}, b_{2}, b_{3}\right)$ represents the number of feedback bits optimally distributed between the base stations.

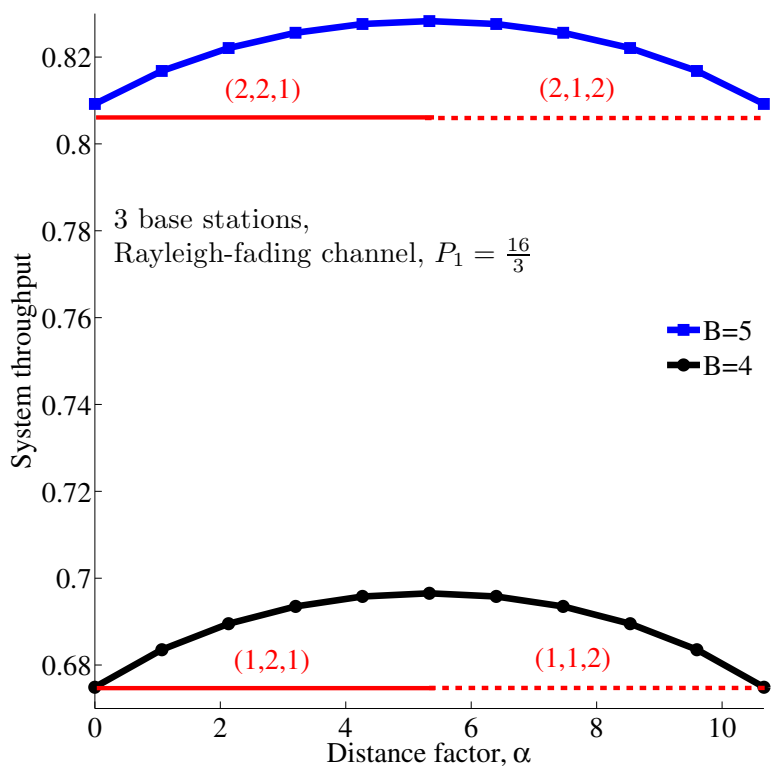

Figure 4. System throughput vs the distance factor $\alpha$ with different number of feedback bits $B$. Rayleigh fading channel, three base stations, $P_{1}=\frac{16}{3}, P_{2}=$ $\frac{32}{3}-\alpha, P_{3}=\alpha$. The triple $\left(b_{1}, b_{2}, b_{3}\right)$ represents the number of feedback bits optimally distributed between the base stations according to variable $\alpha$. 


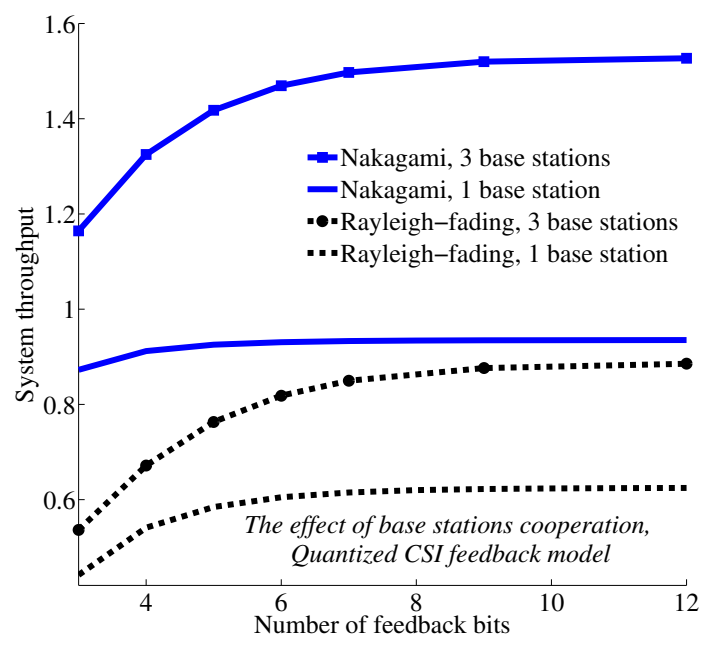

Figure 5. The effect of base stations cooperation on the system throughput. In the case of three base stations, they are assumed to have transmission powers $P_{1}=2, P_{2}=6, P_{3}=8$ and the single base station transmits the data with power $P=16$. For the cooperative base stations, the bit allocation strategy is decided based on Figs. 2 and 3.

- In harmony with practical schemes [8], the simulation results show that in almost all cases the optimal bit allocation matches the one obtained by the Zonal sampling approach [25] (Figs. 2-4). This is a simple and efficient technique which distributes the bits based on the channel variances (here, base stations mapped transmission powers). Starting from the element with highest power, one bit is given to the component with the highest power and then its power is divided by 4 . This procedure continues until all available feedback bits are distributed. Thus, in order to have the highest throughput, the base station with the best channel should get the largest proportion of the feedback bits (Figs. 2-4).

- In all cases, considerable performance improvement is achieved via base station cooperation (Fig. 5). Intuitively, this is due to the fact that in a system with a number of base stations experiencing independent fading conditions it is more likely that, at any time instant, some of the channels experience good channel quality. Therefore, the data transmission efficiency can be improved by always communicating through the best base stations.

\section{CONCLUSiON}

The aim of this paper is to investigate the data transmission efficiency of coordinated multi-point systems in the presence of limited channel quality information feedback. The results are obtained for block-fading channels. It is shown through simulations that considerable performance improvement is achieved via limited number of feedback bits per base station, particularly when the fading is not too severe. The effect of optimal bit allocation becomes more important for the user located in cluster edge areas. Utilizing quantized CSI, the maximum throughput is obtained when the data is transmitted with a rate corresponding to the worst (lowest) gains within the quantization regions, except if the channels fall in their first quantization regions. Moreover, the standard Zonal sampling approach, which allocates a larger proportion of the feedback bits to the best channels, matches the best bit allocation strategy in many cases, maximizing the system throughput. Finally, evaluating the effect of power allocation on the system throughput is left for the future.

\section{REFERENCES}

[1] M. Sawahashi, Y. Kishiyama, A. Morimoto, D. Nishikawa, and M. Tanno, "Coordinated multipoint transmission/reception techniques for LTE-advanced [coordinated and distributed MIMO]," IEEE Wireless Commun. Mag., vol. 17, no. 3, pp. 26-34, June 2010.

[2] M. K. Karakayali, G. J. Foschini, and R. A. Valenzuela, "Network coordination for spectrally efficient communications in cellular systems," IEEE Wireless Commun. Mag., vol. 13, no. 4, pp. 56-61, Aug. 2006.

[3] C. Botella, T. Svensson, X. Xu, and H. Zhang, "On the performance of joint processing schemes over the cluster area," in VTC, May 2010, pp. $1-5$.

[4] J. Li, X. Xu, X. Chen, X. Tao, H. Zhang, T. Svensson, and C. Botella, "Downlink radio resource allocation for coordinated cellular OFDMA networks," IEICE Trans. on Commun., vol. e93-b, no. 12, pp. 34803488, May 2010.

[5] F. Boccardi and H. Huang, "Limited downlink network coordination in cellular networks," in PIMRC, Sept. 2007, pp. 1-5.

[6] A. Papadogiannis, H. J. Bang, D. Gesbert, and E. Hardouin, "Downlink overhead reduction for multi-cell cooperative processing enabled wireless networks," in PIMRC, Sept. 2008, pp. 1-5.

[7] S. Zhou, J. Gong, and Z. Niu, "Distributed adaptation of quantized feedback for downlink network MIMO systems," IEEE Trans. on Wireless Commun., vol. 10, no. 1, pp. 61-67, Jan. 2011.

[8] B. Makki and T. Eriksson, "Efficient channel quality feedback signaling using transform coding and bit allocation," in VTC, May 2010, 1-5.

[9] www.ist-winner.org/.

[10] www.3gpp.org.

[11] G. Caire, G. Taricco, and E. Biglieri, "Optimum power control over fading channels," IEEE Trans. on Info. Theory, vol. 45, no. 5, pp. 1468 $1489,1999$.

[12] R. Knopp, "Coding and multiple access over fading channels," Ph.D. dissertation, Ecole Polytech. Feder. Lausanne, Lausanne, Switzerland, 1997.

[13] N. Arulselvan and R. Berry, "Efficient power allocations in wireless ARQ protocols," in WPMC, vol. 3, Oct. 2002, pp. 976-980.

[14] A. K. Karmokar, D. V. Djonin, and V. K. Bhargava, "Delay constrained rate and power adaptation over correlated fading channels," in GLOBECOM, vol. 6, Nov. 2004, pp. 3448-3453.

[15] H. Seo and B. G. Lee, "Optimal transmission power for single- and multi-Hop links in wireless packet networks with ARQ capability," IEEE Trans. on Commun., vol. 55, no. 5, pp. 996-1006, May 2007.

[16] J. Li, T. Svensson, C. Botella, T. Eriksson, X. Xu, and X. Chen, "Joint scheduling and power control in coordinated multi-point clusters," in VTC, Sept. 2011, pp. 1-5.

[17] B. Makki and T. Eriksson, "On the average rate of quasi-static fading channels with ARQ and CSI feedback," IEEE Commun. Lett., vol. 14, no. 9, pp. 806-808, Sept. 2010.

[18] C. Shen, T. Liu, and M. P. Fitz, "On the average rate performance of hybrid-ARQ in quasi-static fading channels," IEEE Trans. on Commun., vol. 57, no. 11, pp. 3339-3352, Nov. 2009.

[19] G. Caire and D. Tuninetti, "The throughput of hybrid-ARQ protocols for the Gaussian collision channel," IEEE Trans. on Info. Theory, vol. 47, no. 5, pp. 1971-1988, 2001.

[20] T. T. Kim and M. Skoglund, "On the expected rate of slowly fading channels with quantized side information," IEEE Trans. on Commun., vol. 55, no. 4, pp. 820-829, April 2007.

[21] J. G. Proakis, Digital Communications. McGraw Hill, 4th ed., 2001.

[22] B. Sklar, "Rayleigh fading channels in mobile digital communication systems .I. characterization," IEEE Commun. Mag., vol. 35, no. 7, pp. 90-100, 1997.

[23] A. J. Goldsmith and P. P. Varaiya, "Capacity of fading channels with channel side information," IEEE Trans on Info. Theory, vol. 43, no. 6, pp. 1986-1992, Nov. 1997.

[24] B. Makki, L. Beygi, and T. Eriksson, "Channel capacity bounds in the presence of quantized channel state information," Eura. J. on Wireless Commun. and Net., Dec. 2010, doi:10.1155/2010/495014.

[25] K. Sayood, Introduction to Data Compression. Morgan Kaufmann Publishers, 3rd ed., 2006. 